\title{
Edge Impurity Transport Study in Stochastic Layer of LHD and Scrape-off Layer of HL-2A
}

\author{
M. Kobayashi, S. Morita, C.F. Dong \\ National Institute for Fusion Science, Toki 509-5292, Japan
}

Z. Y. Cui, Y. D. Pan, Y. D. Gao, H.Y.Zhou

Southwestern Institute of Physics, P. O. Box 432, Chengdu 610041, China

\author{
Y. Feng \\ Max-Planck-Institute fuer Plasmaphysik, D-17491 Greifswald, Germany
}

S. Masuzaki, M. Goto, T. Morisaki, H. Yamada and the LHD Experiment group
National Institute for Fusion Science, Toki 509-5292, Japan

J. Cheng, P. Sun, Q. W. Yang and X. R. Duan

Southwestern Institute of Physics, P. O. Box 432, Chengdu 610041, China

E-mail contact of main author: kobayashi.masahiro@lhd.nifs.ac.jp

\begin{abstract}
Edge impurity transport has been investigated in the stochastic layer of Large Helical Device (LHD) and the scrape-off layer (SOL) of Huan Liuqi-2A (HL-2A) tokamak, as a comparative analysis based on the three-dimensional (3D) edge transport code EMC3-EIRENE and on the carbon emission profile measurement. The 3D simulation predicts impurity screening effect in the both devices, but also predicts different impurity behavior against collisionality and impurity source location between the two devices. The difference is caused by geometrical structures of the magnetic field lines in the stochastic layer and X-point poloidal divertor SOL, i.e., number of poloidal turns of flux tubes affecting poloidal distribution of plasma parameters and impact of perpendicular transport on parallel pressure conservation and energy transport. These processes have an influence on the impurity screening efficiency at upstream and downstream positions of field lines. The carbon emission measured in the stochastic layer of LHD clearly indicates the screening effect in high density region. The result can be qualitatively interpreted by the present modeling, although the modeling shows a slight difference in the quantitative behavior of carbon ions in the stochastic layer of LHD. On the other hand, comparison of the carbon emission profile from HL-2A with the modeling is not straightforward. It is found that the impurity distribution in the HL-2A SOL is very sensitive to the impurity source location. In order to interpret the experimental observation a further study is necessary, in particular, on the impurity source distribution in the divertor plate and the first wall.
\end{abstract}

PACS: 52.55.Hc, 52.55.Fa, 52.55.Rk, 52.25.Fi, 52.25.Vy

\section{Introduction}

A study of edge impurity behavior is one of critical physics issues in magnetically confined fusion devices. The current topics on the edge impurity behavior are closely related to impurity influx control, detached divertor operation and material migration. In particular, optimization of the heat load on divertor plates, the control of impurity content and the pumping of fuel/helium ash is extremely important as the reactor-relevant issue. Many studies have been attempted with relation to two-dimensional (2D) optimization of the tokamak Xpoint poloidal divertor [1]. On the other hand, three-dimensional (3D) study becomes recently important on the magnetic field geometry such as helical devices $[2,3,4]$ and nonaxisymmetric tokamaks with externally supplied resonant magnetic perturbation (RMP) fields $[5,6,7,8,9,10]$. The $3 \mathrm{D}$ configuration often requires magnetic field stochasticity which brings 
us a totally different edge transport compared to that in the axisymmetric tokamak scrapeoff layer (SOL).

Up to this day the edge impurity transport has been investigated in several magnetic field configurations, e.g., diverted tokamaks $[11,12,13]$, non-axisymmetric tokamaks $[5,14,15]$ and helical devices $[16,17,18,19,20]$. Although the edge impurity transport has been well understood based on such many previous works, the details of the edge impurity transport are still in opened question, in particular, in the combination of parallel and cross-field transports. Therefore, the understanding can be deeper if the edge transport in tokamak scrape-off layer, in which the parallel transport is dominant, is compared with that in helical stochastic layer, in which both the parallel and perpendicular transports are dominant.

Recently, the comparative study between tokamaks and helical devices has been strongly motivated to obtain deeper understanding on the plasma physics. In this paper a comparative study of the edge impurity transport is attempted between different edge magnetic field geometries by comparing the stochastic layer of Large Helical Device (LHD) and the scrapeoff layer of Huan Liuqi-2A (HL-2A) tokamak. For the purpose carbon emission profiles are measured in LHD and HL-2A using extreme ultraviolet (EUV) and vacuum ultraviolet (VUV) spectrometers [21, 22]. The analysis is based on the 3D edge fluid transport code simulation, EMC3 [23] - EIRENE [24], which is implemented in the both devices. An initial result on the comparative study is reported by our co-authors [25]. The present paper describes more detailed analyses on both the modeling and the experiment to provide deeper insight in the transport process between the two devices. We believe that it can clarify the present understanding on the edge impurity transport based on the comparative study with the transport modeling and the current experimental data. We expect that the argument in this paper certainly provides improvements in the future works in the field of edge impurity transport study.

In this paper, the magnetic field structure of LHD and HL-2A is introduced in next section 2. In section 3, the result from 3D edge modeling is presented for both the devices and the difference in the transport between the two devices is also discussed. Section 4 discusses the comparison between the modeling and the measured carbon emission profile. The paper is summarized in section 5 .

\section{Edge magnetic field structures in LHD and HL-2A}

LHD is a heliotron configuration with poloidal and toroidal field periods of $1=2$ and $\mathrm{n}=10$, respectively. The major radius and the averaged minor radius are $3.75 \mathrm{~m}$ and $0.704 \mathrm{~m}$, respectively [2]. The divertor plates on the first wall are made of carbon and the first wall is made of stainless steel. Figures 1 (a), (b) and (c) show the magnetic field structure with last closed flux surface (LCFS) in bird's eye view, the poloidal plasma cross section with divertor configuration in major radius and vertical (R-Z) coordinates and the edge plasma consisting of stochastic region and edge surface layer in the poloidal and effective radius $\left(\theta-r_{\text {eff }}\right)$ coordinates, respectively. The magnetic field connection length $\left(\mathrm{L}_{\mathrm{C}}\right)$ is shown with color bar in Figs. 1(b) and (c). High-temperature plasmas are basically confined without net toroidal current by two helical coils wound around the torus shown in Fig.1 (a). As shown in Fig.1(b), the two helical coils create two X-points at inboard and outboard sides outside the confinement region, and these X-points turn poloidally together with the helical coils when they move toroidally. The four divertor legs created beyond these X-points are connected to the divertor plates. This coil system also creates the stochastic magnetic field structure in the edge region due to the overlapping of magnetic island chains. Some of the flux tubes are stretched out from the X-point and reach the divertor plates, resulting in open field lines. The open field lines near the separatrix of each remnant island constitute magnetic field lines with 
connection lengths longer than $1 \mathrm{~km}$. On the other hand, the open field lines near the O-point of the remnant islands become short flux tubes with lengths shorter than a few tens of meters, as shown with blue and black colors in Figs.1 (b) and (c). Thus, the long and short flux tubes co-exist in the outermost region of LHD, and this region is termed 'edge surface layers' [2]. The inner region with longer $\mathrm{L}_{C}$ is termed 'stochastic region' [2]. Radial extension of these two regions is indicated in Fig. (c). In the present paper, the term of stochastic layer is specifically used to represent the entire edge region of LHD, i.e., outside of LCFS including both the stochastic region and the edge surface layers.

Figure 2 (a) shows a schematic of the HL-2A tokamak with vacuum vessel and divertor chamber/baffles. The HL-2A tokamak has an axi-symmetric X-point poloidal divertor configuration, which is created by three multipole coils as shown in Fig.2 (a). The major radius and the minor radius of HL-2A plasma is $1.65 \mathrm{~m}$ and $\sim 0.4 \mathrm{~m}$, respectively [26]. The divertor plates are made of copper while the baffle and the first wall are made of carbon and stainless steel, respectively. The $\mathrm{L}_{\mathrm{C}}$ distribution in the poloidal cross section is shown in Fig.2 (b). The confinement region with infinite $\mathrm{L}_{\mathrm{C}}$ has a circular shape cross section, surrounded by the scrape-off layer with flux tubes of $\mathrm{L}_{\mathrm{C}} \sim 50 \mathrm{~m}$ in brown colour. Strictly speaking on the LCFS (or separatrix), the $\mathrm{L}_{\mathrm{C}}$ is infinity due to the singularity of the X-point where the poloidal field $\left(B_{\theta}\right)$ is zero. Then, the $\mathrm{L}_{\mathrm{C}}$ rapidly decreases in several millimetres down to the value estimated with $\sim 2 \pi \mathrm{Rq}_{\text {edge. }}$. Here, $\mathrm{q}_{\text {edge }}$ is the safety factor and ranges at $\mathrm{q}_{\text {edge }}=2.8 \sim 4.6$ in the present case. The $\mathrm{L}_{\mathrm{C}}$ then slightly increases outward due to the increase in the safety factor. However, such behaviour of the $\mathrm{L}_{\mathrm{C}}$ is not visible in the Fig. 2 due to the size of figure and also due to the color bar scale set to the same level as that of LHD for comparison. In addition, it seems that the clear separatrix does not exist in reality due to the error field and instead is stochastized to a certain extent.

The divertor has a closed structure with a deep throat in the divertor chamber. The $5 \mathrm{~m}$ long divertor legs are clearly formed below the X-point. The private region is connected to the inner and outer divertor plates with several meters long flux tubes. Parallel distance along the flux tube (those at $\sim 0.01 \mathrm{~m}$ from separatrix at midplane) starting from the outer divertor plate is also denoted with numerals in Fig.2 (b).

Different magnetic field structure between the two devices predicts entirely different edge transport. In the stochastic layer of LHD, the open field lines make a large number of poloidal turns while gradually moving outward due to the radial component of magnetic field, $B_{r} / B_{\phi}=10^{-5} \sim 10^{-3}$. The strong parallel transport distributes the energy in poloidal direction, producing an outward radial flux by replacing the transport into the cross-field direction. Therefore, plasma parameters in the stochastic layer of LHD are almost uniform in the poloidal direction with certain modulation due to the mode structure. A substantial parallel temperature gradient appears in the outside of stochastic layer where the flux tubes are clustered to form the divertor legs. In the SOL of HL-2A, on the other hand, outer and inner divertor plates are directly connected by the flux tubes in one poloidal turn. The parallel transport then becomes dominant to deliver the energy and particles towards divertor plates, and necessarily creates parallel gradient of plasma parameters along the flux tubes. Thus, there appears a strong poloidal asymmetry of plasma parameters in HL-2A.

Important parameters of the two devices are summarized in table 1 to make clear the following explanations.

\section{Energy and impurity transport in the scrape-off layer of HL-2A and in the stochastic layer of LHD}


In subsection 3.1, we first describe the impurity transport model used in the present analysis and the relation between parallel impurity momentum balance and ion energy transport, in addition to the definition of impurity screening or retention. In subsection 3.2 and 3.3, we study the energy transport process in the SOL of HL-2A and in the stochastic layer of LHD, respectively. The results on the modeling analysis are summarized in subsection of 3.4 by comparing the edge transport between the two devices. The operational range of two devices is also given in table 1.

In the present modeling analysis, carbon is adopted as the impurity species, which originates in the divertor plates for the both devices. In this section, the carbon source distributed proportional to the plasma particle deposition pattern is fixed at the divertor plates. Carbon energy released from the divertor plate is fixed to $0.05 \mathrm{eV}$, corresponding to the chemical sputtering. In order to focus on the transport effect of the both devices, we make these assumptions in this section. Other conditions that the first wall is the source of carbon and the carbon is released from the divertor plates with higher energy, which corresponds to physical sputtering, are discussed in section 4 , for detailed comparison with experiments.

\subsection{Impurity transport modeling and definition of impurity screening efficiency}

The 3D edge transport code EMC3 solves the fluid equations for particles, parallel momentum and energies of electrons and ions [23]. The code is coupled with the neutral transport code EIRENE, which solves Boltzmann equation for kinetic transport of atoms and molecules including relevant collisional processes of ionization, recombination and charge exchange etc. [24]. The code is feasible for arbitrary magnetic field and structure of plasma facing components. In the present analysis, a new function is added to the energy transport module of EMC3 to extract the energy flux at each computation cell. This can be done by following the Monte Carlo step on convective and conductive transports at each cell in parallel and perpendicular directions and scoring them in the each cell. The function enables us to extract the heat flux in the convective and conductive energy transports at each cell location including parallel and perpendicular transports, i.e., $q_{/ / V}, q_{/ / D}, q_{\perp V}$ and $q_{\perp D}$.

In the fluid model, the impurity transport along the magnetic field line is expressed by the following classical force balance [27],

$$
m_{z} \frac{\partial V_{z / /}}{\partial t}=-\frac{1}{n_{z}} \frac{\partial T_{z} n_{z}}{\partial s}+Z e E_{/ /}+m_{z} \frac{V_{i / /}-V_{Z / /}^{i m p}}{\tau_{s}}+0.71 Z^{2} \frac{\partial T_{e}}{\partial s}+2.6 Z^{2} \frac{\partial T_{i}}{\partial s}
$$

where $s$ is the coordinate along magnetic field, and the first and second terms on the right hand side express the pressure gradient of impurity and the electrostatic force working on the impurity, respectively. The last three terms originate in the collision with background plasma. The third term is the friction force exerted by the background ion moving with the fluid velocity, $V_{i / /}$, where $\tau_{s}$ is the collision time between impurity and background plasma. The fourth and the fifth terms express the temperature gradient force of electron and ion, respectively, which appears with the parallel temperature gradients and has its origin in the temperature dependence of Coulomb collision, $v \propto T^{-1.5}$. These terms are also called thermal force [28] and directing from low to high temperature region.

The expansion of the impurity pressure gradient term leads to the parallel diffusion of impurity, $\frac{T_{z}}{n_{z}} \frac{\partial n_{z}}{\partial s}=\frac{m_{z}}{n_{z} \tau_{s}} \frac{\tau_{s} T_{z}}{m_{z}} \frac{\partial n_{z}}{\partial s}=\frac{m_{z}}{n_{z} \tau_{s}} D_{/ /} \frac{\partial n_{z}}{\partial s}$ with $D_{/ /} \equiv \frac{\tau_{s} T_{z}}{m_{z}}$, which has a smoothing effect on the density profile, while another term, $\frac{\partial T_{z}}{\partial s}$, is apparently smaller than the ion 
thermal force. The electrostatic force is calculated through the electron momentum equation without current,

$$
\frac{\partial T_{e} n_{e}}{\partial s}+n_{e} e E_{/ /}+0.71 n_{e} \frac{\partial T_{e}}{\partial s}=0
$$

The force usually appears near the divertor plates and often tends to be in opposite direction to the electron thermal force, i.e. directing towards divertor plate. In a steady state condition, the parallel impurity velocity is written as,

$$
V_{Z / /}^{i m p}=V_{i / /}+\frac{\tau_{s}}{m_{z}}\left(-\frac{1}{n_{z}} \frac{\partial T_{z} n_{z}}{\partial s}+Z e E_{/ /}+0.71 Z^{2} \frac{\partial T_{e}}{\partial s}+2.6 Z^{2} \frac{\partial T_{i}}{\partial s}\right)
$$

It is readily found in the present modeling, that the dominant terms on the right hand side of eq.(1) are the friction and the ion thermal forces. The parallel impurity velocity is then given by

$$
V_{Z / /}^{i m p}=V_{i / /}+\frac{2.6 Z^{2} \tau_{s}}{m_{z}} \frac{\partial T_{i}}{\partial s}
$$

Since the background plasma flow, $V_{i / /}$, is usually directed toward the divertor plates, at least near the divertor plates, the flow pushes the impurity downstream through the friction. On the other hand, the parallel temperature gradient is directed upstream. The second term (thermal force) therefore drives the impurity upstream. The resulting impurity velocity is a consequence of the competition between the two terms. Since the term of $\frac{\partial T_{i}}{\partial s}$ indicates upstream flow, the condition of $\left|V_{i / /}\right|<\left|\frac{2.6 Z^{2} \tau_{s}}{m_{z}} \frac{\partial T_{i}}{\partial s}\right|$ also leads to upstream flow for the impurity, suggesting impurity build up at upstream region. Therefore, a necessary condition for avoiding such an impurity build up is given by $\left|V_{i / /}\right|>\left|\frac{2.6 Z^{2} \tau_{s}}{m_{z}} \frac{\partial T_{i}}{\partial s}\right|$. A detailed and systematic treatment of the impurity screening based on the parallel transport eq.(1) is described in ref.[27].

In the direction perpendicular to the local 3D field lines, an impurity is assumed to diffuse with a spatially constant diffusion coefficient, $D_{Z \perp}$, of which the value is equal to all charge states, having the same diffusion coefficient as the background plasma. In the present impurity transport modelling, the impurity is simply smoothed out toward perpendicular direction with the diffusion coefficient of $D_{z \perp}$. It is also noted that [29],

$$
\frac{V_{i / /}}{\frac{2.6 Z^{2} \tau_{s}}{m_{z}} \frac{\partial T_{i}}{\partial s}} \approx \frac{\frac{5}{2} n_{i} T_{i} V_{i / /}}{\kappa_{0}^{i} T_{i}^{2.5} \frac{\partial T_{i}}{\partial s}}=\frac{q_{/ / V}^{i}}{q_{/ / D}^{i}}
$$


where $q_{/ / V}^{i} \equiv \frac{5}{2} n_{i} T_{i} V_{i / /}$ and $q_{/ / D}^{i} \equiv \kappa_{0}^{i} T_{i}^{2.5} \frac{\partial T_{i}}{\partial s}$ are the convection and conduction energy fluxes.

Therefore, the parallel impurity transport is closely related to the ion energy transport in the edge region. In EMC3, these parallel transport terms are calculated not only for the impurity but also for the background plasma in the momentum and energy equations with local 3D coordinates of the stochastic magnetic field. Thus, effect of the radial transport induced by the radial displacement of braiding magnetic field lines is included in this parallel transport modeling.

In the present analysis, the impurity screening is defined as the density fraction of impurity ions which can reach a specific upstream position near the divertor plate, e.g., Xpoint of poloidal divertor tokamaks or LCFS. Thus, degree of the impurity screening can be estimated as $n_{L C F S}^{\text {imp }} / n_{\text {dowm }}^{\text {imp }}$, where $n_{\text {LCFS }}^{\text {imp }}$ and $n_{\text {dowm }}^{\text {imp }}$ are the impurity density at LCFS and near divertor plates, respectively. The state of edge impurity transport can be finally characterized by the ratio, i.e., the impurity screening and the impurity build up are dominant, when $n_{L C F S}^{i m p} / n_{\text {dowm }}^{\text {imp }}<1$ and $n_{L C F S}^{\text {imp }} / n_{\text {dowm }}^{\text {imp }}>1$, respectively.

\subsection{The scrape-off layer of $\mathrm{HL}-2 \mathrm{~A}$}

The reference dataset of HL-2A tokamak for transport simulation are taken from Ohmic discharges with $\mathrm{B}_{\mathrm{t}}=1.42 \mathrm{~T}$, where the operational parameters range in $\bar{n}_{e}=0.75 \sim 4.5 \times 10^{19}$ $\mathrm{m}^{-3}$, I $=150 \sim 250 \mathrm{kA}$ and $\mathrm{V}_{\mathrm{L}}=1.2 \sim 1.5 \mathrm{~V}$ for line-averaged electron density, plasma current and loop voltage, respectively. In these discharges the ohmic heating power ranges from 100 to $400 \mathrm{~kW}$. In the present impurity transport analysis, the value of $200 \mathrm{~kW}$ is selected as a typical SOL input power. The power is equally split into electron and ion energies at the upstream boundary, i.e., LCFS. Because of the limited database in the edge density profile, the density at the LCFS is deduced from the line-averaged electron density as $n_{L C F S}=0.2 \sim 0.3 \bar{n}_{e}$, which gives a reasonable agreement with downstream parameters between the experiment and modeling. The value of $n_{\text {LCFS }}$ is varied in a range of $0.1 \sim 0.7 \mathrm{x}$ $10^{19} \mathrm{~m}^{-3}$ with the fixed Ohmic input power. The perpendicular particle and energy transport coefficients of $D_{\perp}$ and $\chi_{\perp e, i}$ are set to 0.50 and $1.50 \mathrm{~m}^{2} / \mathrm{s}$, respectively. These values are spatially constant in the present modeling. Certainly, these perpendicular transport coefficients can be a function of many parameters such as plasma density, temperature and magnetic field etc [30, 31, 32], as already known as Alcator-scaling, Bohm like or gyroBohm like scalings. In addition, they can vary in space. Unfortunately, however, there are very fewer databases of edge plasma parameters in HL-2A, and thus it is very difficult to discuss the parameter dependence of these transport coefficients. But, at least we have found that the selection of the values, $D_{\perp}=0.50$ and $\chi_{\perp e, i}=1.50 \mathrm{~m}^{2} / \mathrm{s}$, gives a reasonable agreement in the up and downstream plasma parameter change during the density scan, as well as the onset density for detachment transition. Also in the radial profiles of Te and ne we have a reasonable agreement with experiments within the error bars of measurements as shown in Fig.13 in the manuscript. If we set, for example, larger transport coefficients, both the up and downstream temperature become lower and it leads to earlier transition to detachment. As for the radial variation of the transport coefficients, we do see a tendency in Fig. 13 such that the $n_{e}$ profile obtained from the experiments are broader than those from the modelling at the outer radius. This might be attributed to an enhanced convective transport like blobs in the far SOL. For the present modelling, however, we put more emphasis on the region close to LCFS of HL-2A by adjusting the profiles, where most of the impurity populate and play a main role on the transport characteristics. 
In order to discuss the parallel energy transport and to obtain the parallel profile of various quantities, we select the flux tubes of $L_{C}=50 \pm 0.5 \mathrm{~m}$, which correspond to the distance of $0.004 \sim 0.017 \mathrm{~m}$ from the separatrix at outer midplane. The flux tubes in this region carry about $30 \%$ of the energy deposited on the outer divertor plates.

The profiles of parallel energy flux along the flux tubes are plotted in Fig. 3 for $n_{L C F S}$ $=0.3$ and $0.6 \times 10^{19} \mathrm{~m}^{-3}$. The coordinates of $\mathrm{s}$ along field lines are shown in Fig. 2 (b) for the flux tubes of $L_{C}=50 \pm 0.5 \mathrm{~m}$ in the poloidal cross section of HL-2A, where the values of $\mathrm{s}=0$ and $50 \mathrm{~m}$ denote the positions of outer and inner divertor plates, respectively and the values of 5 and $45 \mathrm{~m}$ denote the position of X-point. Negative value of the flux in Fig. 3 represents the direction toward outer divertor plates. The flux has a stagnation point at $\mathrm{s} \sim 20$ $\mathrm{m}$, which corresponds to the top of the torus slightly shifted outwards. The energy flux is dominated by the conduction except in the vicinity of divertor plates. The electron and ion fluxes are almost the same in these flux tubes at low density case in Fig.3(a). They gradually increase as approaching the X-point due to the integration of energy input from LCFS through the perpendicular transport. Below the X-point, the interaction of electrons and ions with deuterium neutrals and impurity (carbon) tends to be significant and the volumetric energy loss through atomic processes reaches 40 to $70 \%$ of the total energy, while some part of the energy is also lost to the private region, e.g., 8 to $18 \%$. These percentages vary with the density. This is the reason for the reduction of the flux toward divertor plates below X-point. In relatively high density case of Fig.3(d), however, the electron energy flux becomes larger than the ion energy flux. This is due to the equilibration process transferring the energy from ions to electrons in the SOL, where the ion temperature is two or three times higher than the electron temperature because of the small parallel heat conductivity of ions.

The terms of eq.(4), $V_{/ /}^{\text {fric }} \equiv V_{i / /}, \quad V_{/ /}^{t h i} \equiv \frac{2.6 Z^{2} \tau_{s}}{m_{z}} \frac{\partial T_{i}}{\partial s}$ are plotted in Figs.3 (b) and (e) with the electron thermal force contribution, $V_{/ /}^{\text {the }} \equiv \frac{0.71 Z^{2} \tau_{s}}{m_{z}} \frac{\partial T_{e}}{\partial s}$, and the parallel electric field contribution, $V_{/ /}^{E} \equiv \frac{\tau_{s}}{m_{z}} Z e E_{/ /}$, in which $\mathrm{Z}=3$ is adopted as a representative charge. In this expression, each term is independent of charge number $Z$ except for $V_{/ /}{ }^{E}$ because of the $\tau_{s} \propto 1 / Z^{2}$ dependence. It is seen that $V_{/ /}^{\text {the }}$ and $V_{/ /}^{E}$ are significantly small compared to others, as mentioned above. The resulting impurity velocity, $V_{z / /}{ }^{i m p}=V_{/ /}^{\text {fric }}+V_{/ /}^{\text {thi }}+V_{/ /}^{\text {the }}+V_{/ /}^{E}$, is shown with thick lines. Because of the dominant ion conduction, the value of $V_{/ /}^{\text {thi }}$ is large in almost entire region except for the vicinity of the divertor plate, where the conduction energy flux is replaced by the convection due to the flow acceleration toward target plates. The friction dominant region of $V_{/ /}^{\text {fric }}>V_{/ /}^{\text {thi }}$, i.e., $V_{z / /}{ }^{i m p}<0$, is restricted to $\mathrm{s}<1 \mathrm{~m}$ at low density, where a substantial fraction of the impurity is ionized beyond this region. The impurity ions are then transported toward upstream due to the strong thermal force and accumulate at upstream of $\mathrm{s}>10 \mathrm{~m}$, as shown in Fig.3 (c). In the high density case, on the other hand, the friction dominant region extends upstream, at least, up to $\mathrm{s} \sim 2 \mathrm{~m}$. The effect mainly comes from the reduction of $\tau_{s}$ related to the increasing collisionality, which even overrides an increase in $\partial T_{i} / \partial s$ and a slight decrease in 
$V_{/ /}^{\text {fric }} \equiv V_{i / /}$. Most of the impurities are ionized in the friction dominant region leading to an effective retention (screening) as shown in Fig.3 (f).

The resulting 2D distributions of impurity density are plotted in Fig.4 (a) for low and high density cases, together with the force balance of $\left|V_{/ /}^{\text {fric }}\right|-\left|V_{/ /}^{\text {thi }}\right|$ in Fig.4 (b). In the figures yellow (or bright) color and blue (or dark) color mean the friction and thermal force dominant regions, respectively. It is seen that most of the scrape-off layer is in the thermal force dominant regime at low density (Fig.4 (b) upper), leading to the significant impurity build up above the X-point (Fig.4 (a) upper). The screening effect near the divertor plates becomes strong and the impurity released from the divertor plates are retained in the divertor when the density increases, as shown in Fig.4 (a) (lower). There remains, however, an upward impurity velocity near the X-point as indicated with the blue color in Fig.4 (b) (lower) even at the high density. This implies less screening effect against the impurity released from the first wall. The details of this effect are discussed in section 4 .

The lower the plasma density is, the closer the SOL is to the convective regime with broader flow profile and the smaller the temperature gradients are, as approaching sheathlimited regime. Therefore, it might give an impression that the friction force becomes large compared to the thermal force. However, what the modelling analysis showed are as follows: here one should also take into account the collision frequency between the impurity and the background plasma flow, which has dependency as $\sim \mathrm{n} / \mathrm{T}^{1.5}$. Absolute value of the flow velocity as well as the temperature gradient does not change so much with the density, i.e., they usually scale almost linearly with the density. However, taking into account the fact that the low density leads to higher SOL temperature, the collision frequency scales with the density much faster than such a linear relation, because of the combined effects of the density in the numerator and the exponent $(=1.5)$ of $\mathrm{T}$ in the denominator. At the low density case, therefore, the collision between the impurity and the background plasma flow becomes significantly small, resulting in a significant decrease in the friction force even if we have a broader flow profile, while reduction of the thermal force is modest. This is the reason why the low density SOL tends to be thermal force dominant.

The energy spectrum and the source location of the neutral impurity have also a critical effect on the impurity transport. In the analysis of this section, the neutral impurity with mono energy of $0.05 \mathrm{eV}$ is assumed with sputtering yield of 0.01 . It is also assumed that the carbon is released from only the divertor plates assuming a carbon deposition layer on the copper plates of HL-2A tokamak, of which the distribution is set to be proportional to the plasma particle deposition pattern. This condition represents the process through chemical sputtering. The physical sputtering is analyzed in section 4. Since the neutral impurity with higher released energy can penetrate deeper in plasmas, it certainly leads to less impurity screening even in high density discharges. It is also indicated in Fig.3 (e) and Fig.4 (b) at s $>2 \mathrm{~m}$ and around X-point, $\left|V_{/ /}^{\text {fric }}\right|<\left|V_{/ /}^{\text {thi }}\right|$, respectively. In this case the impurity ionized above Xpoint, which is originated in the first wall source, is pushed toward the top of torus because of the strong thermal force. As a result, it leads to the impurity buildup around the top of torus (these results are not shown), while it depends on the flow formation mechanism.

The flow acceleration is closely related to the ionization source distribution of background plasma as indicated with the particle conservation equation. In the present model, the divertor recycling is a dominant particle source due to the flux compression in the divertor region. This means that the ionization source is mostly localized in the divertor region as shown later in Fig.9, and the flow acceleration is limited there. A possible flow control scheme has been discussed [33] and demonstrated in the experiment using strong gas puff at upstream. The result leads to an effective impurity screening [34,35] with enhanced flow speed at the upstream region. Recent extensive measurement of the flow profile in tokamak 
scrape-off layer has shown a complex pattern and larger magnitude of upstream flow than expected [36,37]. The flow reversal is observed [38,11,39] even in the case that the friction dominant regime does not lead to the impurity screening. In the present modeling, however, we restrict ourselves to the divertor recycling as the driving mechanism necessary for the edge plasma flow. It is at least a common mechanism for the edge flow formation between tokamaks and helical devices. The analysis then highlights the geometrical effect on the impurity transport, which can give us a good basis for further analysis including more complex processes.

\subsection{The stochastic layer of LHD}

In the present modeling, the simulation is done for magnetic configuration with poloidally averaged radial width of $\sim 10 \mathrm{~cm}$ as the stochastic layer. This corresponds to a magnetic configuration with relatively thick stochastic layer in LHD, e.g., magnetic axis position, $\mathrm{R}_{\mathrm{ax}}$, located at $\mathrm{R}=3.75 \mathrm{~m}$. The discharge in $\mathrm{R}_{\mathrm{ax}}=3.75 \mathrm{~m}$ with $\mathrm{B}_{\mathrm{t}}=2.64 \mathrm{~T}$ is maintained by neutral beam injection (NBI) with input power of 4 to $8 \mathrm{MW}$. The electron density at LCFS, $\mathrm{n}_{\mathrm{LCFS}}$, is varied from 1.5 to $6.0 \times 10^{19} \mathrm{~m}^{-3}$. The perpendicular transport coefficients for particle and energy are fixed to $D_{\perp}=0.50$ and $\chi_{\perp e, i}=1.50 \mathrm{~m}^{2} / \mathrm{s}$, respectively. We have done some analysis on the parameter dependence of perpendicular transport coefficients in ref.[40, 41]. In ref.[40], the constant transport coefficients during density scan have been found to better fit to the experiments compared to those with inverse dependence on density, $D_{\perp} \sim 1 / \mathrm{n}$. In ref.[41], we have found a tendency that the transport coefficient increases with temperature. But the analysis is still in qualitative argument and needs further systematic investigation. Additionally, during the density scan the temperature variation in the stochastic layer is found to be rather small while the density changes substantially. Therefore, the selection of the constant transport coefficients might be appropriate for the present analysis. The argument concerning the spatial variation of the coefficients is given in section 4.1 related to the radial profiles of $T_{e}$ and $n_{e}$ in Fig. 12. For the analysis in this section, the input power is fixed to $8 \mathrm{MW}$.

It is difficult to obtain the parallel profile as done for HL-2A because of the presence of stochastic magnetic field lines in LHD,. Instead, the transport is statistically analyzed for flux tubes in different $\mathrm{L}_{\mathrm{C}}$ values. Namely, the flux tubes are divided into 5 groups depending on the order of $\mathrm{L}_{\mathrm{C}}$, i.e., $\mathrm{L}_{\mathrm{C}}<10 \mathrm{~m}, 10 \leq \mathrm{L}_{\mathrm{C}}<10^{2} \mathrm{~m}, 10^{2} \leq \mathrm{L}_{\mathrm{C}}<10^{3} \mathrm{~m}, 10^{3} \leq \mathrm{L}_{\mathrm{C}}<10^{4} \mathrm{~m}$ and $10^{4}$ $\mathrm{m} \leq \mathrm{L}_{\mathrm{C}}$. The quantity of energy flux is averaged over the flux tubes in each group. The parallel energy flux distribution among different $\mathrm{L}_{\mathrm{C}}$ flux tubes is plotted in Fig.5, for low $\left(\mathrm{n}_{\mathrm{LCFS}}=2.0 \times 10^{19} \mathrm{~m}^{-3}\right)$ and high $\left(5.0 \times 10^{19} \mathrm{~m}^{-3}\right)$ density ranges. In almost all flux tubes, the electron conduction $\left(q_{/ / D}^{e}\right)$ becomes dominant as similarly observed in the HL-2A SOL. At outer radial location, the energy is transferred from long to short flux tubes through perpendicular transport, while the long flux tube usually provides dominant transport channel. In the figures, the values of $r_{\text {eff }}=0.60$ and $0.72 \mathrm{~m}$ correspond to the LCFS and the edge surface layers where the flux tubes are clustered and forming the divertor legs, respectively. In the edge surface layers, the parallel flow acceleration becomes large due to sufficient ionization source as reported in ref. [42]. Due to the acceleration of plasma flow, the energy flux tends to be converted to the convection component $\left(q_{/ / V}^{e}\right.$ and $\left.q_{/ / V}^{i}\right)$ as shown in Figs.5 (c) and (f).

In the low density range $\left(\mathrm{n}_{\mathrm{LCFS}}=2 \times 10^{19} \mathrm{~m}^{-3}\right)$, the ion conduction energy is larger than the convection in most of the region, i.e., $q_{/ / D}^{i}>q_{/ / V}^{i}$, except at $r_{\text {eff }}=0.72 \mathrm{~m}$. The situation $\left(q_{/ / D}^{i}>q_{/ / V}^{i}\right)$ gives rise to the thermal force dominant regime, while the edge surface layers 
are marginally in the friction dominant regime $\left(q_{/ / D}^{i} \leq q_{/ / V}^{i}\right)$. The radial profiles of each velocity contribution to the impurity transport described with eq.(3) are plotted in Fig.6, where the velocity is averaged over poloidal and toroidal directions and the electric field is estimated at $Z=3$ as representative charge state. The positive values represent radially outward velocity. In the low density range, the value of $V_{/ /}^{\text {thi }}$ is larger than $V_{/ /}^{\text {fric }}$ as expected from Figs.5 (a) and (b) except for the edge surface layers, where $V_{/ /}^{\text {fric }} \geq-V_{/ /}^{\text {thi }}$ (Fig.5 (c)) and additional contribution of the electric field leads to the impurity screening. Effect of the parallel electric field in stochastic layer on the impurity transport is also discussed in ref.[43]. However, It is found that the effect is limited only in the low density or low collisionality range, since it diminishes in high density range with high collisionality as shown later in Fig. 6 (c). As a result, in the low density range, the impurity has outward velocity in the edge surface layers resulting in the positive gradient of $n_{\text {imp }}$, as found in Fig. 6 (b). On the contrary, the inward velocity at the stochastic region $\left(\mathrm{r}_{\mathrm{eff}}<0.70 \mathrm{~m}\right)$ caused by the strong ion thermal force gives rise to the impurity buildup around LCFS as shown in Fig. 6 (b).

In the high density range $\left(\mathrm{n}_{\mathrm{LCFS}}=5 \times 10^{19} \mathrm{~m}^{-3}\right)$, the conduction energy flux is entirely reduced, while the convection flux remains almost the same. Most of the flux tubes deliver larger ion convection compared to conduction $\left(q_{/ / D}^{i}<q_{/ / V}^{i}\right)$ except for the longest flux tube near LCFS (See Figs. 5 (d-f)). For electrons, the conduction is still a dominant channel for the energy transport. The thermal force decreases due to reduction of the conduction flux and the friction force is dominant in almost entire stochastic layer. This is clear seeing Fig.6 (c), in which the dominant friction force creates an outward impurity flow. The impurity is effectively screened in the edge surface layers and a positive gradient is also seen in the impurity density, as shown in Fig.6 (d). The impurity buildup is entirely disappeared in the stochastic region $\left(\mathrm{r}_{\mathrm{eff}}<0.70 \mathrm{~m}\right)$ due to the suppression of the thermal force.

The simulated 2D distribution of impurity density is plotted in Fig.7, indicating the force balance of $\left|V_{/ \mid}^{\text {fric }}\right|-\left|V_{/ /}^{\text {thi }}\right|$ between friction and thermal forces. In the figure the friction force dominant region with high impurity density and the thermal force dominant region with low impurity density are expressed with bright colours like yellow and dark colours like blue, respectively. In the low density range $\left(\mathrm{n}_{\mathrm{LCFS}}=2.0 \times 10^{19} \mathrm{~m}^{-3}\right)$, the impurity build up is appeared near the LCFS (see Fig.7 (a)). This is caused by appearance of a strong thermal force, as shown in upper plot of Fig.7 (b) with blue color. With increasing the density, on the other hand, the strong thermal force appeared in the stochastic region begins to be suppressed and replaced by the friction force at high density range $\left(\mathrm{n}_{\mathrm{LCFS}}=5.0 \times 10^{19} \mathrm{~m}^{-3}\right)$, as shown in lower plot of Fig.7 (b). The impurity can be then effectively screened at the edge surface layers. It should be noted that the friction dominant region indicated with yellow and white colors in Fig.7(b) is expanded to all poloidal locations in the periphery region. This implies that the screening is effectively independent of the impurity source location.

\subsection{Comparison of edge impurity transport between $L H D$ and $H L-2 A$}

Non-dimensional parameter of normalized ion collisionality, $v_{S O L}^{* i o n} \equiv l_{/ /} / \lambda_{i i}$, is introduced to compare the edge impurity transport between the two devices. Here, $\lambda_{i i}$ and $l_{/ /}$are the ion collision mean free path and the parallel characteristic length, respectively. The parallel characteristic length can be taken as $\sim L_{C} / 2$ for HL-2A and Kolmogorov length, $L_{K}$, for LHD. The collisional regime for the parallel transport can be then clarified using the 
non-dimensional parameter. The ranges of $v_{S O L}^{* i o n}$ for two devices are given in table 1 . As an index of the screening, the ratio, $n_{\text {LCFS }}^{\text {imp }} / n_{\text {dowm }}^{i m p}$, is plotted in Fig.8 (a) as a function of $v_{S O L}^{*}$, where $n_{L C F S}^{\text {imp }}$ and $n_{\text {dowm }}^{\text {imp }}$ are the impurity densities at LCFS and the vicinity of divertor plates, respectively. The impurity density is calculated by summing up all the charge states of carbon. In HL-2A, $n_{\text {dowm }}^{\text {imp }}$ is calculated by averaging over all flux tubes in the outer and inner divertor legs and $v_{S O L}^{* i o n}$ is evaluated at LCFS. In LHD, the variation of $v_{S O L}^{*_{\text {ion }}}$ in the stochastic layers is indicated with error bars. The highest and lowest values of $v_{S O L}^{*_{i o n}}$ in LHD are obtained from the vicinity of LCFS and the edge surface layers, respectively. The maximum value of $v_{S O L}^{* i o n}$ is taken at almost the highest density range for both devices just before the discharges are switched to the detachment. Higher values of $v_{S O L}^{*_{i o n}}$ in LHD originates in the modest reduction of downstream temperature against the density rise, which is caused by the perpendicular transport as discussed in ref.[17]. As defined in section 3.1, the region of $n_{\text {LCFS }}^{\text {imp }} / n_{\text {dowm }}^{\text {imp }}<1$ in Fig.8(a) means the impurity screening in the present analysis. In addition to the divertor source, the first wall impurity source with uniform distribution is considered to examine the effect of source location, while the background plasma source distribution is unchanged. For the carbon source at the stainless steel first wall, we assume certain amount of carbon deposition layer on the wall. In the figure, the grey and black symbols are for HL-2A and LHD, respectively. Results from the divertor and the first wall sources are indicated with circles and triangles, respectively.

In HL-2A, the ratio of $n_{L C F S}^{i m p} / n_{\text {dowm }}^{i m p}$ with the divertor source rapidly decreases from 3 to 0.1 at $v_{S O L}^{* i o n} \geq 1$ showing a strong impurity screening. On the contrary, any impurity screening does not appear when the impurity source is switched to the first wall. The reason is due to the residual thermal force at the upstream as indicated in Fig.4 (b) with dark blue color around $\mathrm{X}$-point. The thermal force can not be fully eliminated even in the high density range as discussed in the last section. A modeling analysis from larger machines like JET shows that the SOL still has certain screening effect against the first wall source [44]. It is interpreted as due to the increased SOL density, which shifts the impurity ionization location radially outward and thus it can accelerate the parallel transport to the divertor along field lines rather than the cross-field transport enhancing the transport to the core plasma. However, such the effect does not appear in the HL-2A tokamak because the SOL density is inherently low.

The impurity buildup also appears in LHD in the thermal force dominant regime at the lowest collisionality of $v_{S O L}^{* i o n} \sim 1$. However, the ratio of $n_{L C F S}^{i m p} / n_{\text {dowm }}^{\text {imp }}$ is relatively small compared to HL-2A. When $v_{S O L}^{* i o n}$ increases, the ratio goes down to $\sim 0.3$ and saturates at $v_{S O L}^{* i o n} \geq 4$. Therefore, the impurity screening is still effective in LHD even if the impurity source is located at first wall, as shown in Fig.8(a). The behavior of carbon impurity in LHD is very similar between the two different source locations at the divertor and the first wall, in contrast to HL-2A. The reason originates in the distribution of the friction dominant region. Since the friction dominant region in LHD is almost uniformly distributed in the poloidal locations as shown in Fig.7 (b), the impurity screening is effectively available against impurity ions coming from all poloidal directions. Such uniform poloidal distribution of the friction force is attributed to the magnetic field structure in the stochastic layer, where the field lines have 
large number of poloidal turns (several hundred turns) before they reach divertor plates. Therefore, plasma parameters tend to be distributed uniformly in the poloidal direction. In the case of HL-2A tokamak, on the other hand, the field lines in SOL have only one poloidal turn. This gives rise to the poloidal asymmetry of plasma parameters as shown in Fig.4 (b).

It is also important to note a difference in the ionization distribution of background plasmas between the two devices because it is a main mechanism of the flow acceleration appeared in the present modeling. The ionization source is limited below the X-point in HL2A, as shown in Fig.9 (b).

In LHD, the divertor plates are positioned along the divertor legs rotating in the poloidal direction, of which the position is uniquely determined by the helical coil geometry, as shown in Fig.1. Therefore, the recycling neutrals from the divertor plates in LHD exist in all the poloidal directions. The spread neutral distribution is also an important key feature to provide the poloidally uniform friction dominant region mentioned above. As discussed in the last section, however, the mechanism of the flow formation might alter the result, besides the ionization source. In particular, the effect is emphasized in recent experimental observation in the tokamak devices [36].

It is also found for the divertor source case in Fig.8(a) that the ratio of $n_{L C F S}^{\text {imp }} / n_{\text {dowm }}^{\text {imp }}$ in HL-2A is largely reduced at higher $v_{S O L}^{* i o n}$ compared to that in LHD, suggesting a strong impurity screening effect of HL-2A. This is attributed to different values of $q_{/ / V}^{i} / q_{/ / D}^{i}$ between two devices, as shown in Figs. 8 (b) and (c). In the figures, the downstream is defined as $\mathrm{s}=0 \sim 0.5 \mathrm{~m}$ (see Fig.2(b)) for HL-2A and $\mathrm{r}_{\mathrm{eff}}=0.72 \mathrm{~m}$ for LHD (see Fig.1(c)), i.e., the vicinity of divertor plates, while the upstream is defined as $\mathrm{s}=3 \sim 8 \mathrm{~m}$ for HL-2A, i.e., the vicinity of X-point, and $r_{\text {eff }}=0.65 \mathrm{~m}$ for LHD, i.e., the stochastic region. The impurity screening for the divertor source is mainly relevant to $q_{/ / V}^{i} / q_{/ / D}^{i}$ at downstream. The value of $q_{/ / V}^{i} / q_{/ / D}^{i}$ at downstream in HL-2A increases ten times with increasing $v_{S O L}^{* i o n}$, while that in LHD is entirely modest keeping nearly constant values of $2 \leq q_{/ / V}^{i} / q_{/ / D}^{i} \leq 4$. The difference can be explained by taking into account the perpendicular transport. In the tokamak SOL, a strong coupling between upstream and downstream expressed by $T_{\text {down }} \propto n_{u p}{ }^{-2}$ and $n_{\text {down }} \propto n_{u p}{ }^{3}$ exists in the limited conduction regime based on the pressure conservation [27]. These relations imply a strong decrease in $q_{/ / D}^{i}$ in the downstream region, since $q_{/ / D}^{i} \propto T_{\text {down }}{ }^{2.5} \propto n_{\text {up }}{ }^{-5}$. Here, we neglect the change of $\partial T_{i} / \partial s$ for the simplicity. The change of $q_{/ / V}^{i}$ is not so large in the frame of the present fluid modeling because of a priori assumption in Bohm boundary condition at the divertor plate, i.e., $q_{/ / V}^{i} \propto n_{\text {down }} T_{\text {down }}^{1.5} \propto n_{\text {up }}{ }^{3} n_{\text {up }}{ }^{-3} \sim$ constant. From this assumption, one might expect a strong increase in the energy flux ratio of $q_{/ / V}^{i} / q_{/ / D}^{i}$ with increasing density. This gives a favorable effect to the impurity screening.

In LHD, on the other hand, the coupling between upstream and downstream along the flux tubes becomes weaker in the stochastic layers, that is, $T_{\text {down }} \propto n_{\text {up }}{ }^{-1 \sim-2 / 3}$ and $n_{\text {down }} \propto n_{\text {up }}{ }^{1 \sim 1.5}$ [17], since the perpendicular transport is enhanced. Then, it gives much more modest dependence on the upstream density compared to the tokamak SOL. Based on the same argument as in HL-2A, the energy flux through ion conduction is written by $q_{/ / D}^{i} \propto T_{\text {down }}^{2.5} \propto n_{u p}{ }^{-5 / 3 \sim-2.5}$, if the change of $\partial T_{i} / \partial s$ is neglected. The change of $q_{/ / V}^{i}$ does not seem to be so large, since the energy flux through ion convection, 
$q_{/ / V}^{i} \propto n_{\text {down }} T_{\text {down }}^{1.5} \propto n_{\text {up }}^{1 \sim 1.5} n_{u p}{ }^{-1 \sim 1.5}$, is almost constant. Here, one can expect the enhancement of $q_{/ / V}^{i} / q_{/ / D}^{i}$ at the downstream with increasing density. But the value in LHD is smaller than that in the HL-2A tokamak SOL. This is the reason why the impurity screening is modest at the downstream in LHD compared to HL-2A.

Suppression of the thermal force is discussed in ref.[16] based on the perpendicular energy transport enhanced by the island divertor of W7-AS. Here, the same effect is also considered in the stochastic region of LHD with many remnant small islands (see Fig.1 (c)). Radial energy flux in the stochastic region can be expressed as

$$
q_{r e, i}=-n \chi_{\perp e, i} \frac{\partial T_{e, i}}{\partial r}-\vec{b} \cdot \vec{r} \kappa_{0 e, i} T_{e, i}^{2.5} \frac{\partial T_{e, i}}{\partial s}
$$

in the poloidal cross section of stochastic layer. The symbol of $\vec{r}$ denotes the base vector in radial direction, where only the conduction energy is considered for simplicity. The first and second terms of the right hand side represent the perpendicular and $\left(q_{\perp}\right)$ and parallel contribution $\left(q_{/ /}\right)$, respectively. Using $\frac{\partial}{\partial r}=\frac{1}{\vec{b} \cdot \vec{r}} \frac{\partial}{\partial s}$, the ratio of the parallel to perpendicular transports is written as,

$$
\frac{q_{\|}}{q_{\perp}}=\frac{\Theta^{2} \kappa_{0 e, i} T_{e, i}^{2.5}}{n \chi_{\perp e, i}}
$$

where $\Theta \equiv \vec{b} \cdot \vec{r} \approx B_{r} / B_{\phi}$. In LHD, the value of $\Theta \equiv \vec{b} \cdot \vec{r} \approx B_{r} / B_{\phi}$ is estimated to be $10^{-4}$ to $10^{-3}$ for ions. For typical edge plasma parameters in LHD, e.g. $100 \mathrm{eV}$ and $10^{19} \mathrm{~m}^{-3}$, these values give a condition of $q_{/ /}<q_{\perp}$ for ions. It is noted here that the ion energy flux is relevant for the impurity transport as discussed in section 3.0. This means that the $q_{/ /}$is replaced by the enhanced $q_{\perp}$, which may be called "bypass effect", resulting in the suppression of parallel ion conduction energy, $q_{/ / D}^{i}$, in other words, suppression of the thermal force. This effect becomes large in high density and low temperature ranges, as seen in eq.(8). It also brings a condition of $q_{/ / V}^{i} / q_{/ / D}^{i} \geq 1$ in the stochastic region, as shown in Fig.8 (c). Suppression of the thermal force in the stochastic region reinforces the screening effect at high $v_{S O L}^{* i o n}$ range in LHD. The modest decrease in the downstream temperature in LHD also leads to detachment transition in higher density range. This is favorable for increasing $q_{/ / V}^{i} / q_{/ / D}^{i}$ through the "bypass effect" given by eq.(8).

If we apply the same argument to HL-2A SOL, the eq.(7) can be rewritten as

$$
q_{\theta e, i}=-n \chi_{\perp e, i} \frac{1}{r} \frac{\partial T_{e, i}}{\partial \theta}-\vec{b} \cdot \vec{\theta} \kappa_{0 e, i} T_{e, i}^{2.5} \frac{\partial T_{e, i}}{\partial s}
$$

in the poloidal cross section. The symbol of $\vec{\theta}$ denotes the base vector in poloidal direction. Using $\frac{1}{r} \frac{\partial}{\partial \theta}=\frac{1}{\vec{b} \cdot \vec{\theta}} \frac{\partial}{\partial s}$ and $\Theta \equiv \vec{b} \cdot \vec{\theta} \approx B_{\theta} / B_{\phi}$, one can obtain the eq.(8). For HL-2A 
tokamak, the value of $\Theta \equiv B_{\theta} / B_{\phi}$ ranges in $10^{-2} \sim 10^{-1}$. A condition of $q_{/ /} / q_{\perp}>>1$ is obtained from the eq.(8) for typical edge plasma parameters in HL-2A. Therefore, the "bypass effect" of energy flux by the perpendicular transport is not available in HL-2A tokamak. This is one of the reasons why the $q_{/ / V}^{i} / q_{/ / D}^{i}$ can not be brought to above unity at upstream even in high $v_{S O L}^{*_{i o n}}$ range of HL-2A, in contrast to LHD (see Fig.8 (b)). This can be also confirmed by the presence of residual thermal force around X-point in high density range (see Fig.3 (e) and Fig.4 (b)).

The above arguments based on the simulation are summarized in the following.

1. Edge impurity screening effect is compared between two devices of HL-2A and LHD using the ratio of $n_{\text {LCFS }}^{\text {imp }} / n_{\text {dowm }}^{\text {imp }}$ as a function of normalized ion collisionality, $v_{S O L}^{*^{i o n}}$. The $v_{S O L}^{* \text { ion }}$ takes different values between two devices because of different contribution of the perpendicular transport (ref.[17]).

2. HL-2A has a strong impurity screening effect when the impurity source is located at divertor, while no screening effect is observed when the impurity source is switched to the first wall. LHD has a sufficient impurity screening effect against both the divertor and the first wall impurity sources. However, the (reduced value of $n_{L C F S}^{i m p} / n_{\text {dowm }}^{\text {imp }}$ against the divertor impurity source in LHD indicates a relatively modest impurity screening compared to HL-2A.

3. Different screening effect against the divertor impurity source between the two devices is attributed to the coupling of plasma pressure between upstream and downstream, i.e., $T_{\text {down }} \propto n_{\text {up }}{ }^{-2}$ and $n_{\text {down }} \propto n_{\text {up }}{ }^{3}$ for HL-2A and $T_{\text {down }} \propto n_{\text {up }}{ }^{-1 \sim-2 / 3}$ and $n_{\text {down }} \propto n_{\text {up }}{ }^{1 \sim 1.5}$ for LHD. These dependences can affect the ratio of $q_{/ / V}^{i} / q_{/ / D}^{i}$ (=friction force/thermal force), i.e., $q_{/ / V}^{i} / q_{/ / D}^{i}>10$ for HL-2A and $q_{/ / V}^{i} / q_{/ / D}^{i} \sim 5$ for LHD.

4. Effective impurity screening against the first wall source in LHD is caused by the poloidally uniform distribution of the friction dominant region, which is originated in a large number of poloidal turns of field lines in the stochastic layer before reaching divertor plate. In HL-2A SOL, on the other hand, the fiction dominant region is poloidally asymmetric. It is only dominant near the divertor plates because the flux tube in HL-2A tokamak poloidally rotates only one time during one toroidal turn. The impurity screening is also affected by the ionization distribution of the background plasma. The flow acceleration derived in the present modeling effectively works for the impurity screening.

5. Perpendicular energy flux can largely contribute to the impurity screening in the stochastic region of LHD, in which the parallel energy flux is replaced by the perpendicular one. It finally leads to the suppression of the thermal force at upstream. This contribution certainly reinforces the impurity screening effect in high $v_{S O L}^{* i o n}$ range. In addition, the impurity screening can be also assisted by increasing a threshold density triggering the plasma detachment based on the modest coupling between upstream and downstream in the stochastic layer. It can thus work to extend the operational range toward higher $v_{S O L}^{*_{i o n}}$ range. 


\section{Measurement of carbon line emissions in LHD and HL-2A and interpretation by numerical modeling}

In this section, chord-integrated intensity and radial profile of carbon line emissions measured by VUV and EUV spectrometers are analyzed with the modeling as a function of density. Effects of different impurity source location and released energy of carbon atom are discussed with experimental data from the two devices. In subsection of 4.1, experimental setup, selection of the emission lines, numerical setup of the background plasma and examination of the impurity source characteristics are described. In subsections of 4.2 and 4.3 , the results are discussed for LHD and HL-2A, respectively.

\subsection{Experimental and numerical setup}

VUV and EUV spectrometers have been installed on LHD [21,45] and HL-2A [22] to investigate the edge impurity transport. The viewing areas of the VUV and EUV spectrometers in LHD are shown in Figs.10 (a) and (b), respectively. The area covers the divertor legs and the X-point as well as the peripheral region of the plasma. The obtained line emission can thus reflect the behavior of carbon impurity ions through the downstream to upstream in the stochastic layer. The viewing area of the VUV spectrometer in HL-2A shown in Fig. 20 (a) covers the lower half of the plasma including marginally the X-point, while the divertor legs are outside of the observation angle. The obtained emission thus provides information through the mid-stream to upstream in the SOL of HL-2A.

The ionization stages of $\mathrm{C}^{2+}$ and $\mathrm{C}^{3+}$ ions are measured as CIII (977 $\AA$ : $\left.1 \mathrm{~s}^{2} 2 \mathrm{~s}^{2}-1 \mathrm{~s}^{2} 2 \mathrm{~s} 2 \mathrm{p}\right)$ and CIV (1548 $\left.\AA: 1 \mathrm{~s}^{2} 2 \mathrm{~s}-1 \mathrm{~s}^{2} 2 \mathrm{p}\right)$ for both devices, respectively, while $\mathrm{C} 4+$ ions are differently measured, i.e., CV (40.27 $\left.\AA: 1 \mathrm{~s}^{2}-1 \mathrm{~s} 2 \mathrm{p}\right)$ for LHD and $2271 \AA \AA$ : $1 \mathrm{~s} 2 \mathrm{~s}-1 \mathrm{~s} 2 \mathrm{p}$ for HL-2A. The emission coefficients of these lines are found to be almost independent of the electron density in the range of $\mathrm{n}_{\mathrm{e}}=10^{18} \sim 10^{20} \mathrm{~m}^{-3}$. Therefore, we can utilize the relation between the carbon emission and the plasma parameters, $I^{Z}\left(T_{e}, n_{e}\right)=n_{i m p}{ }^{Z} n_{e} L^{Z}\left(T_{e}\right)$, where $Z$ is the charge state and $L^{Z}\left(T_{e}\right)$ the emission coefficient. The $L^{Z}\left(T_{e}\right)$ are plotted in Fig. 11. It is found that the emission coefficient of CIII and CIV, which have the ionization potential, $\mathrm{E}_{\mathrm{i}}$, of 48 and $65 \mathrm{eV}$ respectively, are almost independent of the temperature in the range $10 \sim 100 \mathrm{eV}$. This temperature range is most relevant in the present analysis. This means that the intensity measured from $\mathrm{C}^{2+}$ and $\mathrm{C}^{3+}$ ions is directly related to the impurity density, if it is divided by $\mathrm{n}_{\mathrm{e}}$. The emission coefficient of CV $\left(\mathrm{E}_{\mathrm{i}}=392 \mathrm{eV}\right)$, on the other hand, significantly depends on the temperature as shown in the Fig.11. Therefore, the interpretation of the CV should take into account this temperature dependence, as discussed later.

Figure 12 shows $T_{e}$ and $n_{e}$ profiles at the outer midplane of LHD measured by Thomson scattering system, together with the modelling results. The LCFS is estimated at around $\mathrm{R}=4.55 \mathrm{~m}$. In the 3D transport computation of EMC3, the perpendicular transport is assumed to be anomalous, while classical transport is assumed for the parallel transport. The perpendicular transport coefficient of particle and energy, $D_{\perp}$ and $\chi_{\perp e, i}$, are assumed to be spatially constant and selected to well fit the $\mathrm{T}_{\mathrm{e}}$ and $\mathrm{n}_{\mathrm{e}}$ measured in the experiment.

In Fig.12 (a), the modelling results with different $\chi_{\perp e, i}$ 's are shown for fixed input power. When we use the value of $\chi_{\perp e, i}=1.50 \mathrm{~m}^{2} / \mathrm{s}$, we have a good agreement between the modelling and the experiment at $\mathrm{R}>4.65 \mathrm{~m}$, while there is a substantial deviation at $\mathrm{R}<4.65$ $\mathrm{m}$. On the other hand, we have a better fit to the experiment by taking smaller $\chi_{\perp e, i}$ at $\mathrm{R}<$ $4.65 \mathrm{~m}$, while the profile starts to deviate at $\mathrm{R}>4.65 \mathrm{~m}$. Since we use a spatially constant value, $\chi_{\perp e, i}$, the result indicates a change of $\chi_{\perp e, i}$ around $\mathrm{R}=4.65 \mathrm{~m}$. Concerning the impurity screening, the absolute value of temperature plays an important role on the force balance in the impurity momentum transport. In the present analysis, we have found that the 
qualitative change of the force balance mainly occurs at the outer region as shown in Fig.6. In the inner region, on the other hand, there is no qualitative change because the thermal force tends to always persist although its magnitude changes with density. For the present analysis, therefore, we have decided to take the profile with $\chi_{\perp e, i}=1.50 \mathrm{~m}^{2} / \mathrm{s}$ as shown in Fig. 12,

which gives the best fit to Te at the outer region and thus enables us to analyse qualitative behaviour of impurity transport. However, it is certainly important to have a better fit in the inner region for more precise and quantitative argument of impurity transport. For this purpose, we are now improving the code to be capable of having spatially varying the transport coefficients. This is left as the future work.

For the density profile, we have found that the value of $D_{\perp}=0.50 \mathrm{~m}^{2} / \mathrm{s}$ gives a reasonable agreement with the measurement as shown in Fig. 12 (b). The value is found to be almost independent of the density, as discussed in section 3.3. The region of $\mathrm{R}<4.65 \mathrm{~m}$, where there always exists a systematic deviation of $\mathrm{T}_{\mathrm{e}}$ between the modeling and the experiment as mentioned above, corresponds to the temperature range relevant to $\mathrm{C}^{4+}(\mathrm{CV})$. Radial profiles of the emission coefficient for each charge state estimated from the Thomson $\mathrm{T}_{\mathrm{e}}$ are plotted in Fig. 12 (c). The CV profile simulated with the 3D model is also plotted in the figure. The modeling result shows a smaller $\mathrm{CV}$ emission by a factor of $2 \sim 3$ compared to the emission from the experiment.

The radial profiles of $T_{e}$ and $n_{e}$ at the outer midplane of HL-2A obtained by a scanning probe [46] are plotted in Fig. 13, together with the modeling result. In the 3D modeling, the transport coefficients are selected as $D_{\perp}=0.50 \mathrm{~m}^{2} / \mathrm{s}$ and $\chi_{\perp e, i}=1.50 \mathrm{~m}^{2} / \mathrm{s}$ and fixed during the density scan. The location of LCFS is determined by analyzing the phase change of $\mathrm{k}_{\theta}$ spectrum [46] and the uncertainty in the determination is estimated to be $\pm 0.5 \mathrm{~cm}$. The probe data show a relatively slow radial decay for both the parameters, in particular, in the far SOL region. This might be attributed to the non-diffusive transport. Within the error bars of the measured parameters and the positional uncertainty, the result of the modeling fairly agrees with the experimental profiles.

In the present modeling, we examine four different impurity sources, i.e., $0.05 \mathrm{eV}$ and $2 \mathrm{eV}$ carbon energies released from the divertor plates and the first wall. As mentioned in the last section, the first wall carbon source is assumed with certain amount of carbon deposition layer on the stainless steel surface of the first wall. The divertor plate source is distributed being proportional to the plasma particle deposition pattern, while the first wall source is assumed to be uniform. The $0.05 \mathrm{eV}$ released energy represents the chemical sputtering with fixed sputtering coefficient of 0.01 , while the $2 \mathrm{eV}$ released energy supposes the physical sputtering, in which the sputtering coefficient changes depending on the incident particle energy [47].

The chemical erosion depends on many parameters such as ion flux [48], surface temperature [49] and energy of the projectile [50]. We have examined the data concerning these parameters obtained in the both devices as well as the database of sputtering rate reported in the references. It is, however, rather difficult to estimate accurately the erosion rate from such comparison between the experimental data and the reported databases because the both databases have substantial scatter. It is also noted that the erosion rate depends on the surface condition as well, for which we have almost no information. We certainly need systematic experimental study on the sputtering rate for more accurate and quantitative arguments of the analysis, which is currently underway by improving diagnostics and left for future work. Taking into account the parameter dependences and also the scatter in the database of the chemical sputtering rate in ref. [48,49,50], the best estimation of the chemical sputtering rate would be an order of 0.01 with error bar of a factor of $\sim 2$. Instead of addressing the erosion process directly, on the other hand, it might be reasonable to check the 
behaviour of CIII emission, since it is usually considered to mostly reflect the impurity source rate, not being affected by the transport so much due to the low charge state. In this respect, the density dependence of CIII in Fig.17 (experiment) and 18 (e) (modelling) for LHD shows a reasonable agreement between the two within a factor of $\sim 2$, which roughly corresponds to the error bar expected in the estimation of chemical erosion rate. In other words, this is the best result we can do at the moment for the qualitative analysis based on the limited data concerning chemical erosion rate. As for HL-2A, we find a discrepancy between the experiment and the modelling concerning the behaviour of CIII, as shown later. The reason is discussed including the selection of chemical sputtering rate and other effects in section 4.3.

The absolute recycling flux on the divertor plate calculated in the modeling has showed a reasonable agreement with the experiment [40]. The charge-exchange (CX) neutral flux to the first wall estimated from the simulation depends on the divertor flux. The first wall flux is comparable to the divertor flux in LHD, whereas the first wall flux is one order of magnitude less than the divertor flux in HL-2A taking into account the closed divertor configuration. The chemical and physical sputtering have a comparable contribution to the impurity source in the low density range, while the chemical sputtering is dominant in the high density range because the physical sputtering yield decreases with reduction of the edge temperature, i.e., reduction of the incident energy [47].

\subsection{Results of LHD}

Figures 14 (a) and (b) show the $\mathrm{C}^{3+}$ density distribution obtained from the modeling with the chemical sputtering on divertor plates in LHD for different density ranges of $\mathrm{n}_{\mathrm{LCFS}}=1.5$ and $5.0 \times 10^{19} \mathrm{~m}^{-3}$, respectively. The input power to the stochastic layer is changed from $4 \mathrm{MW}$ at the low density to $8 \mathrm{MW}$ at the high density, according to the NBI deposition power. Results from other carbon source cases also show a similar distribution to Fig.14, while a deeper penetration of carbon is observed in the case of physical sputtering on the first wall. The modeling shows that the edge region is entirely dominated by the thermal force in the low density, but it changes to the friction dominant regime in the high density. When the thermal force is dominant, it is found that the $\mathrm{C}^{3+}$ ions deeply penetrate in the stochastic region and distribute around the poloidal mode structures of $\mathrm{m}=4$ and 5 , where the distribution becomes almost uniform in the poloidal direction as seen in Fig.14 (a). In the high density case, on the other hand, the increased friction force pushes the carbon ions radially outward, and the $\mathrm{C}^{3+}$ ions populate around the mode structures of $\mathrm{m}=3$ and 4 with higher rotational transform. In this case the poloidal modulation of the $\mathrm{C}^{3+}$ density becomes large, resulting in the localized distribution around the divertor legs and the top and bottom of the LHD plasma, as shown in Fig.14 (b).

The change against the density is reflected on measured profiles of impurity line emissions in LHD. Upper half vertical profile of CIV (1548 $\AA$ ) line emission is shown in Fig. 15 as the typical example. It is clearly seen that the CIV in the high density discharge at $\mathrm{n}_{\mathrm{LCFS}}=4.5 \times 10^{19} \mathrm{~m}^{-3}$ increases around the midplane $(\mathrm{Z}=0.04 \mathrm{~m})$ and the top of torus $(\mathrm{Z}=0.48 \mathrm{~m})$, respectively. The measured CIV profiles are analyzed with the present simulation code.

Profiles obtained from the modeling are plotted in Figs. 16 (a) - (d) for different impurity source locations (divertor and first wall) and different released energies $(0.05$ and $2.0 \mathrm{eV})$. The profile summed up over all the sources is plotted in Fig. 16 (e), where the contribution of the chemical sputtering is dominant at the high density. With increasing density, the CIV profile becomes peaked around $\mathrm{Z}=0.05 \mathrm{~m}$ and $0.47 \mathrm{~m}$, as shown in Fig. $16(\mathrm{e})$. The peaked profile is caused by the localization of $\mathrm{C}^{3+}$ density at the divertor legs and the top of torus as shown in Fig. 14 (b). It is found that the profiles from high density cases show a similar result in the appearance of the peaks even if the impurity sources are different. 
However, the shape in the first wall source case is flatter around $Z=0.05$ and $0.47 \mathrm{~m}$ compared to divertor source case. This is clearly seen in Fig.16, e.g. comparing Fig.16 (a) and (b) or Fig.16 (c) and (d), respectively. This is due to less screening effect against the first wall source compared to the divertor source. It is attributed to the poloidal modulation of stochastic layer thickness, i.e., it is thicker at the divertor leg region (the region of out- and inboard midplane in Fig.1 (b)) and thinner in front of the first wall (the top and bottom of the plasma in Fig.1 (b)). The neutral carbon coming from the first wall, e.g., from the top and the bottom of torus, can penetrate closer to the LCFS because the stochastic layer is thinner, compared to the thicker layer at the in- and out-board sides, which can effectively stop the neutral carbon from the divertor. Since the friction dominant region is mainly formed at the peripheral region (edge surface layers) as shown in Fig.6 (c) and Fig.7 (b), the carbon ionized at deeper location has less chance to feel the friction force. Therefore, the screening effect becomes weak against the first wall carbon source, and thus it leads to a flatter CIV profile as shown in Figs.16 (b) and (d).

The simulated profile in Fig.16(e) can be compared with the experimental profile in Fig.15. The experimental profile also shows a few clear peaks at some vertical locations similar to the modeling prediction. The result suggests the impurity movement from the upstream to the downstream region with increasing density, which can be understood by the enhancement of the friction force.

The correlation between the impurity line measurement and the modeling indirectly supports the identification of magnetic field structure in plasmas. A clearer evidence has been reported with $\mathrm{H}_{\alpha}$ and impurity emission measurements in Tore Supra [51,52] and LHD [53] and divertor power load measurement in TEXTOR-DED [54].

The density dependence of the carbon emission observed in the experiment is plotted in Fig. 17, where the intensity is normalized by the electron density. Due to the different ionization potential of carbon ions, i.e., CIII $(48 \mathrm{eV}), \mathrm{CIV}(65 \mathrm{eV})$ and $\mathrm{CV}(392 \mathrm{eV})$, the intensities of CIII and CIV are interpreted as a proxy for the carbon source and CV as a proxy for the impurity located at deeper radial position. The interpretation of CIII and CIV is straightforward due to the almost constant emission coefficient in the range of 10 to $100 \mathrm{eV}$, as shown in Fig. 11. Namely, the change of CIII and CIV intensities represents the change of $\mathrm{C}^{2+}$ and $\mathrm{C}^{3+}$ ion densities, respectively. Both intensities indicate a constant carbon influx against the line-averaged density. The reduction of $\mathrm{CV}$ intensity indicates the impurity screening. The modeling is studied to explain the experimental result in details.

Density dependence of the carbon line emissions in each charge state is studied by the 3D modeling. The result is shown in Fig.18, where the intensity is normalized by the electron density. In all the cases with different sources, the emission from high charge state (CV) clearly shows a different density dependence compared to those from low charge states (CIII and CIV). If we assume only the chemical sputtering (see Figs.18 (a) and (b)), the intensities of CIII and CIV increase with density while the CV first decreases with density but starts to increase at $n_{L C F S}=3 \times 10^{19} \mathrm{~m}^{-3}$. No difference is seen between the divertor and the first wall sources with chemical sputtering. This is due to the poloidally distributed friction force dominant region as discussed in section 3.4 and in Fig. 7 (b). In the case of physical sputtering (see Figs.18 (c) and (d)), the CIII and CIV intensities slightly decrease with density and then seems to saturate at $\mathrm{n}_{\mathrm{LCFS}}=4 \times 10^{19} \mathrm{~m}^{-3}$, whereas the CV intensity monotonically decreases with density, mainly reflecting the sputtering coefficient decreasing with density increase.

The summation of these different sources is plotted in Fig. 18 (e), where the CIII and CIV tend to increase with density while the CV decreases with density until it saturates at $\mathrm{n}_{\mathrm{LCFS}}=4.0 \times 10^{19} \mathrm{~m}^{-3}$. It is found that the physical sputtering contributes to the emission only at the low density, where it has a comparable flux with the chemical sputtering. A deeper 
penetration of physically sputtered impurity provides larger radiation due to the high plasma density at the inner radius. With increasing density, the contribution of the physical sputtering is replaced by the chemical one, which starts to dominate in the amount of carbon influx. Thus, the density dependence at the high density range roughly follows the result of chemical sputtering. When the experimental result in Fig. 17 is compared with the modeling result in Fig.18(e), we notice that the contribution from the chemical sputtering is considerably overestimated in the modeling. In LHD, the emission from hydrocarbon molecule is usually weak except for extremely high density discharges with strong hydrogen gas puffing at $\mathrm{n}_{\mathrm{e}}>10^{20} \mathrm{~m}^{-3}$. This experimental result, which is different from general feature appeared in tokamaks, may be caused by low operational density range (order of $10^{18} \mathrm{~m}^{-3}$ ) in open divertor system of LHD. Since the ion temperature in divertor region is higher than tokamak due to the low divertor density, the self-physical sputtering by carbon can be possibly enlarged [55].

The carbon density profiles for the case combined all sources (Fig.18 (e)) are plotted in Fig.19. The total carbon density summed over all charge states $\left(\mathrm{n}_{\mathrm{imp}}\right)$ changes from peaked to hollow profile due to the impurity screening effect in the high density. All charge states of carbon shift their radial position outward with density. The peak density in low charge states of carbon $\left(\mathrm{C}^{2+}\right.$ and $\left.\mathrm{C}^{3+}\right)$ increases, while that in high charge state $\left(\mathrm{C}^{4+}\right)$ slightly decreases. Taking into account the temperature dependence of the emission coefficient of CV in Fig.11, the decrease in $\mathrm{CV}$ observed in the modeling is due to a combined effect of the reduction of $\mathrm{C}^{4+}$ density and the outward shift to low temperature region.

The impurity screening in a stochastic layer with the friction force has also been predicted in previous theoretical analyses [56,57,58,59,60,61]. Although some of them also combine an effect of the parallel electric field, it is not found to be so significant in the present modeling. A similar experimental observation of the impurity screening with stochastic magnetic field is also reported in TEXT [62], Tore Supra [14] and TEXTOR-DED [15]. The present analysis consolidates these predictions and observations, and adds further insight into the transport process by analyzing the 3D modeling including a precise magnetic field structure of the stochastic layer. It should be noted here that other mechanisms which can influence the impurity screening have to be considered for further study on the impurity transport in the stochastic layer. For example, spatially different perpendicular transport and perpendicular electric field $[63,64]$ are the candidate as the alternative mechanism. However, those are not included in the present modeling due to the difficulty in the formulation.

\subsection{Results of $\mathrm{HL}-2 \mathrm{~A}$}

A similar assumption on the impurity source characteristics is also used for analyzing the edge impurity transport in HL-2A except for the first wall impurity source. The first wall source which is one order magnitude less than the divertor source is adopted in the simulation, since the neutral flux at the first wall above X-point in HL-2A is significantly suppressed by the closed divertor configuration. The $2 \mathrm{D}$ distribution of $\mathrm{C}^{3+}$ density in HL-2A obtained by the modeling is plotted in Fig. 20 for two different densities, where the impurity source is located at the divertor plates proportional to the plasma particle deposition pattern with fixed sputtering coefficient of $\mathrm{C}_{\text {sput }}=0.01$ and with released energy of $0.05 \mathrm{eV}$. The line integrated CIV profiles are measured with the viewing area shown in Fig. 20 (a). The result is plotted in Fig. 21 for different line averaged densities. The $\mathrm{Z}$ coordinate starts from the upper boundary of the viewing area and increases downward, as indicated in Fig. 20 (a). In the modeling the density at LCFS, $\mathrm{n}_{\mathrm{LCFS}}$, is related to the line averaged density by $n_{\mathrm{LCFS}}=0.2 \sim 0.3 \bar{n}_{e}$. The measured CIV profile becomes flat in the low density range, while it becomes gradually peaked at around $\mathrm{Z}=0.35 \mathrm{~m}$ with increasing density. The profiles are analyzed using the present modeling in details as follows. 
The line integrated CIV profiles obtained in the modeling using the viewing area shown in Fig.20 (a) are plotted in Fig. 22, for different source characteristics. At the low density of $\mathrm{n}_{\mathrm{LCFS}}=0.3 \times 10^{19} \mathrm{~m}^{-3}$, the line integrated CIV profiles become flat in the all source cases. This is due to the strong thermal force, which drives the impurity upward, and the most of the $\mathrm{C}^{3+}$ are populated above the X-point as shown in the 2D distribution of Fig. 20 (a). This impurity buildup at the upstream always occurs in the low density case, independent of the source location and the released energy. At the high density, however, the CIV profile is significantly different depending on the impurity source characteristics. With increasing density, the friction force near the divertor plates becomes strong and the thermal force at upstream is weakened. Most of the carbon atoms released from the divertor is retained near the divertor plate as ions in lower charge states of $\mathrm{C}^{+}$and $\mathrm{C}^{2+}$. The resultant $\mathrm{C}^{3+}$ distribution is then localized around X-point and the density decreases, as shown in Fig. 20 (b), indicating that the divertor impurity source is dominant. The line integration of CIV emission is therefore peaked around $\mathrm{X}$-point near $\mathrm{Z}=0.40 \mathrm{~m}$ and the intensity decreases as seen in Fig. 22 (a). Such a behavior becomes different for the first wall source. No peaking is seen in the CIV profile and the intensity increases with density, as shown in Fig. 22(b). That is to say, the tendency is completely opposite to the divertor source case. This is due to the residual thermal force at the upstream as discussed in section 3.4 and in Fig.4 (b). The residual thermal force leads to the impurity buildup for impurity ions entering the SOL above X-point even at the high density. The impurity density increases almost proportional to the source amount. A similar effect of the thermal force working at the entrance to the outer divertor leg, which drives impurity ions upward, has been observed in the modeling analysis in JET [11].

In the case of physical sputtering, the CIV intensity decreases in the high density range for both the impurity sources of divertor and first wall, as shown in Figs. 22 (c) and (d), respectively. This is due to the reduction of sputtering coefficient with increasing density at which the incident energy of impinging particles decreases. The effect is stronger than the upward drive force of impurity ions based on the residual thermal force above the X-point. The resulting CIV profile combining the all impurity source cases is plotted in Fig. 22(e). The behavior of CIV is not a simple function of the density. It decreases first and increases at the higher density. The profile tends to peak at the edge region at the highest density.

When the modeling (Fig.22(e)) is compared with the experimental result in Fig.21, we notice that the density dependence of CIV intensity is quite different each other, whereas the tendency of profile change with small peak near the X-point is similar between the experiment and the modeling in the high density region. The CIV intensity in HL-2A simply increases with density, which is not reproduced in the modeling shown in Fig. 22(e). Since the present transport modeling of the HL-2A tokamak suggests that the impurity distribution is sensitive to the impurity source location, the identification of the source characteristics is important for understanding the experimental result. In order to make better explanation of the experimental results, other impurity source cases are tested in the modeling. The sputtering coefficients increasing with density is adopted for the divertor impurity source, as shown in Fig.23(a), and a new impurity source located at the baffle is considered with fixed sputtering coefficient of 0.01, as shown in Fig.23(b). The newly introduced impurity source shows a better agreement with the experimental result not only in the intensity but also in the profile. At present there is no clear reason why the sputtering coefficient increases with density at the moment. However, the plasma interaction with the baffle is probably one of possible candidates to explain the experimental result because the position of plasma edge sometimes has a discrepancy between the experiment and calculation using EFIT code. It seems that the experimental result observed here can be explained as a combination of several impurity sources assumed in the modeling. Further study is necessary for these topics. 
The density dependence is also studied by measuring carbon emissions in different ionization stages. The result is plotted in Fig. 24. Although the data are a little scattered reflecting a small difference among discharges of HL-2A, the CIII and CIV emissions seem to be nearly constant against the line averaged density, while the CV emission clearly shows a gradual reduction against the density. The density dependence of these emissions is analyzed with the modeling. The result is plotted in Figs. 25 (a) - (d) for different impurity sources as used in Fig.22. The intensity of the all charge states decreases with increasing density for the divertor source case, as shown in Figs. 25 (a) and (c). This is due to the strong screening effect below the X-point. On the other hand, in the case of the first wall source with the fixed $\mathrm{C}_{\text {sput }}=0.01$ shown in Fig. 25 (b), the CIII and CIV increase with increasing density, while the $\mathrm{CV}$ strongly decreases. The increase in CIII and CIV is attributed to the impurity buildup above X-point which occurs even at the high density as discussed above. Although such the difference of screening effect due to the source location is identified only in the modeling in the present analysis, the similar tendency has been identified in the methane puff experiments in JET [44]. The decrease in CV is caused by a limited computational domain of the inner boundary which is set to several $\mathrm{cm}$ inside the LCFS. In HL-2A, the edge temperature is considerably low and it changes from $200 \mathrm{eV}$ to $100 \mathrm{eV}$ in such a location during the density scan. The radial location of CV moves further inside the plasma core when the density is increased. Thus, the $\mathrm{CV}$ is mostly located out of the computational domain, in particular, at high density discharges of HL-2A. Therefore, it inhibits a direct comparison of the CV emission obtained in the modelling with experiments. The CV emission is affected by the dependence of life time in each charge state on plasma parameters in addition to the perpendicular transport in the core region, rather than the parallel force balance between the friction and thermal force. However, such analyses are beyond the scope of the present investigation. In the following, therefore, the discussions are focused on the behaviour of CIII and CIV in comparison with experiments, although the $\mathrm{CV}$ obtained from the modelling is included in the following figures. In the case of physical sputtering, all the emissions decrease with density increase. This is due to the diminishing sputtering coefficient in the high density range, independent of the source location.

The density dependence of these emissions combining all the different sources is plotted in Fig. 25 (e). It shows that the CIII and CIV intensities in low charge states slightly decrease in higher densities, while the $\mathrm{CV}$ intensity in high charge state continuously decreases with density.

The roughly constant CIII and CIV intensities against the density scan observed in the experiment (see Fig.24) indicate a little difference from the modeling in higher density range (see Fig.25 (e)). It simply tells us the importance of the chemical sputtering at the first wall because the wall chemical sputtering predominantly determines the CIII and CIV intensities at the high density range as shown in Figs.25 (a)-(d). The decrease in measured CV intensity has a similar tendency to the modelling, but it is quantitatively different. The reduction of $\mathrm{CV}$ in the modeling is roughly two orders of magnitude, while the reduction in the modeling is only a factor of 3. The CV intensity in the modeling is entirely underestimated by the limited computational domain as mentioned above.

The same analysis is also done with other impurity sources shown in Fig. 23. The result is plotted in Fig. 26. As expected from Fig. 23, the dependence of CIII and CIV intensities shows a little better agreement with the experiment, whereas the reduction of CV is still much larger than the experiment due to the same reason as mentioned above. Detailed understanding on the edge impurity behavior in HL-2A is, however, still difficult.

In HL-2A, it is found that the result is very sensitive to the impurity source location, in contrast to the result from LHD. The difference comes from geometrical effect of the 
magnetic field structure on the impurity transport as discussed in section 3.4. The analysis shows an importance of identification of the impurity source characteristics for interpretation of experimental results in HL-2A. In order to make further development on this study, quantitative comparison between the modeling and experiment is necessary in addition to precise measurement on edge spectral line profile with its absolute emissivity. These are left as the future work. Finally, it should be noted that the comparison of edge impurity behavior between helical and tokamak plasmas has provided us a deeper insight into the impurity transport property.

\section{Summary}

LHD and HL-2A have a distinct edge magnetic field structure in viewpoints of the connection length and the flux tube topology. In order to study the effect of magnetic field geometry on the edge impurity transport, a comparative transport analysis has been conducted between LHD stochastic layer and HL-2A SOL. For this purpose, the 3D edge transport code of EMC3-EIRENE has been implemented and the profile measurement of carbon emissions has been performed using EUV and VUV spectrometers in the both devices.

Comparison of the simulation results shows a clear difference in the impurity screening process between LHD and HL-2A. HL-2A SOL has a strong screening effect against the divertor impurity source at high density with high collisionality. However, no impurity screening is appeared when the impurity source is replaced by the first wall. Because the residual thermal force still effectively works at the upstream above the X-point even at the highest density just before the attached plasma changes to the detached one. The LHD stochastic layer has a sufficient impurity screening effect against both the divertor and first wall sources. The effect is however weaker compared to the divertor impurity source case in HL-2A SOL.

The effective impurity screening against the first wall source in LHD observed in the modeling is due to the presence of the poloidally distributed friction dominant region formed by a large number of poloidal turns (several hundred turns) of field lines in the stochastic layer before they reach the divertor plate. In HL-2A, on the other hand, one poloidal turn flux tube creates a poloidally asymmetric friction dominant region located only in the vicinity of the divertor plate. The ionization distribution of background plasma is also closely related to these different magnetic structures. The acceleration mechanism of background plasma and thus impurity flow along the magnetic field line is basically determined by the ionization distribution in the present modeling. However, further analysis is necessary on the flow formation, especially in the tokamak SOL. Since it is recently reported that the background plasma is significantly accelerated even at the midplane, the result might change the picture obtained in the present modeling.

The enhanced perpendicular transport in the stochastic layer is found to affect the impurity transport in two ways. First, the upstream region changes in the friction dominant regime, which is usually impossible in the tokamak SOL. This is due to the enhanced perpendicular energy transport, which can replace the parallel ion conduction energy flux ("bypass effect"), i.e. reduces the thermal force. Second, the impurity screening effect at downstream is weakened compared to the tokamak SOL. The effect is attributed to the perpendicular loss of parallel momentum in the stochastic flux tubes. resulting in relatively high temperature and low density at downstream. Both are caused by the geometrical effect of magnetic field in the stochastic layer.

Comparison of the modeling result with measured CIV line emission profile in LHD shows a qualitative agreement. The density dependence of CIII, CIV and CV intensities also qualitatively shows a good agreement with the modeling. It should be pointed out here that 
the profile of CIV emission is not sensitive to the impurity source location due to the presence of poloidally distributed friction dominant region, as mentioned above. The agreement with the experimental result can be interpreted as downstream movement of carbon $\mathrm{C}^{3+}$, indicating the impurity screening effect in high density region.

In the case of HL-2A, interpretation of the experimental data is not straightforward. The experimental result can be reasonably explained, if it is possible that the sputtering coefficient increases with density or the baffle is the impurity source instead of the divertor plate. It should be also pointed out that the edge impurity distribution in HL-2A tokamak is very sensitive to the impurity source location, in contrast to LHD. It is important to identify the source characteristics from the experimental study in order to make a consistent interpretation of the impurity transport.

\section{Acknowledgements}

The authors wish to thank the LHD and HL-2A teams for their excellent cooperation. The authors are grateful for the support for the computational resources: Plasma Simulator SR16000 L2 powered by HITACHI Ltd, and AMD Opteron-InfiniBand Cluster Machine, by Grant-in-Aid for Science Research on Priority Areas (Area 465, No.18070005) from MEXT Japan. The computational work is partly supported by the NIFS budget code NIFS10ULPP016. This work was partly supported by the JSPS-NRF-NSFC A3 Foresight Program in the field of Plasma Physics (NSFC: No.11261140328). 


\section{References}

[1] A Loarte et al., "Progress in the ITER physics Basis: Chapter 4 : Power and particle control”, Nucl. Fusion 47 (2007) S203.

[2] Ohyabu N. et al., Nucl. Fusion 34 (1994) 387.

[3] Grigull P. et al., Plasma Phys. Control. Fusion 43 (2001) A175.

[4] Strumberger E., Nucl. Fusion 36 (1996) 891.

[5] Ghendrih Ph et al., Nucl. Fusion, 42 (2002) 1221.

[6] Finken K.H. et al., Nucl. Fusion 47 (2007) 522.

[7] Eich T. Reiser D. and Finken K.H. Nucl. Fusion 40 (2000) 1757.

[8] Abdullaev S.S. et al., Nucl. Fusion 43 (2003) 299.

[9] Evans T. et al., Nature Phys. 2 (2006) 419.

[10] H. Frerichs et al., Nucl. Fusion 52 (2012) 023001.

[11] Strachan J. D. et al, Nucl Fusion 48 (2008) 105002.

[12] Shimizu K. et al., Nucl. Fusion 49 (2009) 065028.

[13] Petrie T.W. et al., Nucl. Fusion 49 (2009) 065013.

[14] Corre Y. et al., Nucl. Fusion 47 (2007) 119.

[15] Telesca G. et al., J. Nucl. Mater. 390-391 (2009) 227.

[16] Feng Y. et al., Nucl. Fusion 46 (2006) 807.

[17] Kobayashi M. et al., Fusion Sci. Technol. 58 (2010) 220.

[18] M.B.Chowdhuri et al. Physics of Plasmas 16 (2009) 062502.

[19] H.Nozato, S.Morita and M.Goto, Review of Scientific Instruments 76 (2005) 073503.

[20] C.F.Dong, S.Morita, M.Goto and M.Kobayashi, Plasma Science and Technology 13 (2011) 140.

[21] Dong C.F. et al., Rev. Sci. Instrum. 81 (2010) 033107.

[22] Cui Z. et al., Rev. Sci. Instrum. 81 (2010) 043503.

[23] Feng Y. et al., Contrib. Plasma Phys. 44 (2004) 57.

[24] Reiter D. et al., Fusion Sci. Technol. 47 (2005) 172.*

[25] Feng Y. et al., Plasma Phys. Control. Fusion 53 (2011) 024009.

[26] Yang Q.W. et al., Nucl. Fusion 47 (2007) S635.

[27] Stangeby P.C. "The plasma boundary of magnetic fusion devices", Institute of Physics Publishing, Bristol (2000).

[28] Braginskii S.I. "Review of plasma physics " vol.1 p.205, M. Leontovich, Ed., Consultants Bureau, New York (1963).

[29] Igitkhanov Y. Contrib. Plasma Phys. 28 (1988) 477.

[30] T. Ohkawa, Physics Letters 67A (1978) 35.

[31] B. LaBombard, J.A. Goetz et al., J. Nucl. Mater. 241-243 (1997) 149.

[32] A. Loarte, S. Bosch et al., J. Nucl. Mater. 266-269 (1999) 587.

[33] Burrell K.H., Phys. Fluids 19 (1976) 401.

[34] Schaffer M.J. et al., J. Nucl. Mater. 241-243 (1997) 585.

[35] Itami K. et al., J. Nucl. Mater. 266-269 (1999) 1097.

[36] Asakura N. and ITPA SOL and Divertor Topical Group, J. Nucl. Mater. 363-365 (2007) 41.

[37] Takizuka T. et al., Nucl. Fusion 49 (2009) 075038.

[38] Nedospasov A.V., Tokar M.Z., 11th EPS Conf. on Controlled Fusion and Plasma Physics, Aachen, 1983, Vol.7F, Part 2, p.21.

[39] Cooke P.I.H. and Prinja A.K., Nucl. Fusion 27 (1987) 1165.

[40] Y. Feng et al., Nucl. Fusion 48 (2008) 024012.

[41] S. Masuzaki, M. Kobayashi, T. Morisaki et al., J. Nucl. Mater. 390-391 (2009) 286.

[42] M. Kobayashi et al., Contrib. Plasma Phys. 48 (2008) 255. 
[43] Hsu J.Y., Harvey R.W. and Wong S.K., Phys. Fluids 24 (1981) 2216.

[44] J.D. Strachan et al. Nucl. Fusion 43 (2003) 922.

[45] M.Goto, S.Morita, et al., Fusion Science ans Technology 58 (2010) 394.

[46] Cheng, J. et al., Nucl. Fusion 49 (2009) 085030.

[47] M. Kobayashi et al., "Study on impurity screening in stochastic magnetic boundary of the Large Helical Device", Proceedings of 22nd IAEA FEC (13-18th Oct. 2008, Geneva), EX/9-4.

[48] J. Roth, A. Kirschner et al., J. Nucl. Mater. 337-339 (2005) 970.

[49] B.V. Mech, A.A. Haasz and J.W. Davis, J. Nucl. Mater. 255 (1998) 153.

[50] J.W. Davis and A.A. Haasz, J. Nucl. Mater. 241-243 (1997) 37.

[51] Nguyen F., Ghendrih P. and Grosman A., Nucl. Fusion 37 (1997) 743.

[52] Hogan J. et al., Plasma Phys. Control. Fusion 44 (2002) 673.

[53] Dong C.F. et al., Phys. Plasmas 18 (2011) 082511.

[54] Lehnen M. et al., Plasma Phys. Control. Fusion 47 (2005) B237.

[55] W. Eckstein et al., "Sputtering Data”, IPP 9/82, (1993) Max-Planck-Institut fuer

Plasmaphysik.

[56] Dei-cas R. and Samain A., Plasma Physics and Controlled Nuclear Fusion Research 1 (1975) 563.

[57] Shishkin A.A., Nucl. Fusion 21 (1981) 603.

[58] Samain A., Grosman A. and Feneberg W., J. Nucl. Mater. 111-112 (1982) 408.

[59] Samain A. et al., J. Nucl. Mater. 128-129 (1984) 395.

[60] Nicolai A., Schoengen F. and Reiter D., Plasma Physics 27 (1985) 1479.

[61] Tokar M.Z. et al., Plasma Phys. Control. Fusion 39 (1997) 569.

[62] Rowan W.L. et al., in Proceedings of 14th EPS conference, Control. Fusion and Plasma Physics 11D (1987) 117.

[63] Oren L. et al., J. Nucl. Mater. 111-112 (1982) 34.

[64] Braun S. and Helander P., Journal of Physics: Conference Series 260 (2010) 012004.

*http://www.eirene.de/ 